\title{
Commentary Combinatorial Psycho-Pharmacological Approaches for the Treatment of Abnormal Aggression
}

\author{
Sam A Golden ${ }^{*, 1}$ and Aki Takahashi, ${ }^{*}$ \\ 'Neurobiology of Relapse Section, National Institute on Drug Abuse, Baltimore, MD, USA; ${ }^{2}$ Laboratory of Behavioral Neuroendocrinology, University \\ of Tsukuba, Tsukuba, Japan
}

Neuropsychopharmacology (2018) 43, 233-234; doi:I0.1038/npp.20 17.174; published online II October 2017

Aggression is a highly evolutionarily adaptive behavior that is critical for survival and reproduction, and therefore, strongly ethologically conserved across species. However, due to environmental or biological processes, aggressive behavior can transition from adaptive to maladaptive. This transition is associated with detrimental outcomes for both the individual exhibiting the behavior and their victims. Within the United States, an estimated 2.7 million individuals over the age of 12 report experiencing one or more violent victimizations annually (Truman and Morgan, 2016), and $\sim 70 \%$ of victims of serious violence experience ongoing socio-emotional problems as a direct result (Langton and Truman, 2014). Because of the marked impact violence has on societal health and well-being, there have been efforts to identify the underlying risk factors associated with the development of abnormal aggression. Longitudinal prospective population-based studies have consistently associated future violence and crime with early-life adversities such as abuse or neglect. Strikingly, early-life neglect is one of the most damaging predisposing factors, even more so than inter-personal violence and physical or sexual abuse (Gilbert et al, 2009).

Analogous to human behavior, aggression in rodent models is typically assessed as either species-typical or atypical abnormal (escalated) aggression (Miczek et al, 2013). When adaptive, rodent aggression most often occurs within the contexts of competing for essential resources or defending territory and offspring. Such species-typical aggressive behaviors are highly stereotyped, generally consisting of non- or low-injurious bites directed at an intruder's back and flanks that cease when an intruder displays submissive signals. Conversely, escalated aggression is

*Correspondence: Dr SA Golden, Neurobiology of Relapse Section, Intramural Research Program, National Institute on Drug Abuse, 25 I Bayview Boulevard, Suite 200, Baltimore, MD 21224, USA, Tel: +44 3740 2613, Fax: +443 740-2827, E-mail: sam.golden@nih.gov or Dr A Takahashi, Laboratory of Behavioral Neuroendocrinology, University of Tsukuba, Research Building D4I8, I-I-I Tennoudai, Ibaraki, Tsukuba 305-8577, Japan, Tel: + 81-298-53-2499, E-mail: akitakahashi@kansei.tsukuba.ac.jp

Received 3 August 2017; accepted 6 August 2017 defined by prolonged, frequent injurious bites aimed at vulnerable body parts such as the head, throat, paws, and underbelly (Miczek et al, 2013). Previously, Toth et al (2008) reported that prolonged post-weaning early-life social isolation in rats' results in escalated aggressive behavior against intruders. Importantly, this social isolation occurs during a critical juvenile developmental window, a period characterized by heightened neural plasticity and representing a critical period for the programming effects of social behavior on the brain (Tzanoulinou and Sandi, 2017). Therefore, prolonged post-weaning social isolation in rodents is proposed to model components of social neglect in humans.

In this issue of Neuropsychopharmacology, Mikics et al (2017) tested whether a combinatorial strategy, using both behavioral re-socialization and pharmacological manipulation to reactivate critical window-like plasticity, will decrease abnormal aggression following social isolation. Specifically, they proposed that fluoxetine, an anti-depressant that has been shown to reactivate critical period like plasticity within the amygdala (Karpova et al, 2011), would synergistically reactivate plasticity for social learning and allow resocialization to exert restorative anti-aggressive effects.

As previously reported, escalated aggression was observed in rats subjected to prolonged early-life social isolation, but not to socially reared rats. While neither re-socialization nor fluoxetine alone were effective, the combination of both approaches decreased escalated aggressive behavior. On the basis of previous studies implicating the reinitialization of critical window-like plasticity by fluoxetine on brain-derived neurotrophic (BDNF) factor signaling, the authors performed targeted transcriptional profiling of BDNF transcripts in aggression-related brain regions. Social isolation decreased $B D N F$ transcript in all regions assayed, but was only rescued in the infralimbic region of the medial prefrontal cortex (mPFC) following both co-administration of re-socialization and fluoxetine, but not either independently. In contrast, chronic fluoxetine, but not resocialization itself, decreased methylation on the promotor region of $B D N F$ exon 4 within in $\mathrm{mPFC}$. In addition, pharmacological inhibition of the BDNF receptor, $\operatorname{TrkB}$, in 
mPFC abolished the effect of combinatorial re-socialization and fluoxetine treatment on escalated aggression, while pharmacological activation of TrkB signaling combined with re-socialization successfully suppressed abnormal aggression. Together, these data suggest that $\operatorname{TrkB}$ activation in $\mathrm{mPFC}$ mimics the effect of chronic fluoxetine treatment, allowing for increased plasticity and re-learning of species-typical social behavior.

These findings represent a significant advance to our understanding of how social neglect influences the onset of abnormal aggression, and more importantly, point to a novel therapeutic strategy for treatment. Clinically, the effects of anti-depressant treatments on aggressive behavior have been controversial and variable. Some studies showed a reduction of aggression, while others find no effect or even increased aggression following chronic fluoxetine treatment (Sharma et al, 2016). Similarly, behavioral therapies based on enhanced socialization show, at best, a modest effect on aggression in individuals who were exposed to early-life adversities (Currie and Startup, 2012). Mikics et al (2017) proposed that aggression treatment should include both approaches for greatest efficacy. Illustratively, in adult mice social isolation models, fluoxetine treatment can effectively reduce the duration of aggressive behavior, while the current data demonstrate that early-life social isolation is insensitive to fluoxetine or re-socialization when administered separately. This exemplifies that early-life social isolation is more deleterious and requires more complex treatments.

In sum, the findings of Mikics et al (2017) have translational potential and will hopefully instigate mechanistic studies that emphasize the importance of combinatorial approaches to the treatment of complex social behaviors. Additional research is now required to better understand the downstream effectors of BDNF signaling in $\mathrm{mFPC}$ following early-life adversity. Surprisingly, a short-term pharmacological administration of either a $\operatorname{TrkB}$ activator or inhibitor was sufficient to modulate the effect of re-socialization. A question for future research is the mechanism underlying such a fast action and whether this treatment causes plasticity within the local microcircuit or through re-wiring of $\mathrm{mPFC}$ afferent projections from other brain regions such as the ventral hippocampus (vHPC). The authors conclude their manuscript with the exciting finding that combinatorial therapy increased the number of vHPC neurons projecting to the mPFC. Future functional studies are necessary to determine whether this projection is a candidate for therapeutic investigation.

\section{FUNDING AND DISCLOSURE}

The authors declare no conflict of interest.

\section{ACKNOWLEDGMENTS}

We thank Dr Yavin Shaham for insightful comments on a draft of this manuscript. The research was supported by the Intramural Research Program of NIDA and a National Institute of General Medical Sciences Postdoctoral Research Associate Grant 1FI2GM117583-01 (SAG), and KAKEN 17H04766, 15K12773 (AT).

\section{REFERENCES}

Currie M, Startup M (2012). Doing anger differently: two controlled trials of percussion group psychotherapy for adolescent reactive aggression. J Adolesc 35: 843-853.

Gilbert R, Widom CS, Browne K, Fergusson D, Webb E, Janson S (2009). Burden and consequences of child maltreatment in highincome countries. Lancet 373: 68-81.

Karpova NN, Pickenhagen A, Lindholm J, Tiraboschi E, Kulesskaya N, Agustsdottir A et al (2011). Fear erasure in mice requires synergy between antidepressant drugs and extinction training. Science 334: 1731-1734.

Langton LL, Truman JL (2014). Socio-emotional impact of violent crime. Bureau of Justice Statistics. Available at: https://www.bjs. gov/index.cfm?ty $=$ pbdetail\&iid $=5114$.

Miczek KA, de Boer SF, Haller J (2013). Excessive aggression as model of violence: a critical evaluation of current preclinical methods. Psychopharmacology 226: 445-458.

Mikics E, Guirado R, Umemori J, Toth M, Biro L, Miskolczi C et al (2017). Social learning requires plasticity enhanced by fluoxetine through prefrontal Bdnf-TrkB signaling to limit aggression induced by post-weaning social isolation. Neuropsychopharmacology. (Advance online publication, 9 August 2017; doi:10.1038/ npp.2017.142).

Sharma T, Guski LS, Freund N, Gotzsche PC (2016). Suicidality and aggression during antidepressant treatment: systematic review and meta-analyses based on clinical study reports. BMJ 352: 165.

Toth M, Halasz J, Mikics E, Barsy B, Haller J (2008). Early social deprivation induces disturbed social communication and violent aggression in adulthood. Behav Neurosci 122: 849-854.

Truman JL, Morgan RE (2016). Criminal victimization, 2015. Bureau of Justice Statistics. Available at: http://www.bjs.gov/ index.cfm?ty $=$ pbdetail\&iid $=5804$.

Tzanoulinou S, Sandi C (2017). The programming of the social brain by stress during childhood and adolescence: from rodents to humans. Curr Top Behav Neurosci 30: 411-429. 